\title{
Quality evaluation of mycelial Antrodia camphorata using high-performance liquid chromatography (HPLC) coupled with diode array detector and mass spectrometry (DAD-MS)
}

Sandy Shuo Zhao, Kelvin Sze-Yin Leung ${ }^{*}$

\begin{abstract}
Background: Antrodia camphorata (AC) is an important fungus native to Taiwanese forested regions. Scientific studies have demonstrated that extracts of AC possess a variety of pharmacological functions. This study aims to identify the full profile fingerprint of nucleosides and nucleobases in mycelial AC and to assess the quality of two commercial mycelial AC products.

Methods: High-performance liquid chromatography coupled with diode array detector and mass spectrometry was employed to identify the major components in mycelial AC. The chemical separation was carried out using a gradient program on a reverse phase Alltima $\mathrm{C}_{18} \mathrm{AQ}$ analytical column $(250 \times 4.6 \mathrm{~mm}, 5 \mu \mathrm{m})$ with the mobile phase consisting of deionized water and methanol.

Results: Ten nucleosides and nucleobases, two maleimide derivatives, and a sterol were identified as the major constituents in mycelial AC. These groups of chemical compounds constitute the first chromatographic fingerprint as an index for quality assessment of this medicinal fungus.
\end{abstract}

Conclusions: This study provides the first chromatographic fingerprint to assess the quality of mycelial AC.

\section{Background}

Antrodia camphorata (M. Zang \& C.H. Su) Sheng H. Wu, Ryvarden \& T.T. Chang (Polyporaceae) is a parasitic fungus on decayed wood or the inner wall of the heartwood of Cinnamomum kanehirai hay, a tree endemic to Taiwan. Before Antrodia camphorata (AC) was first officially classified as a species in 1990, its medicinal value had been greatly appreciated for many decades. This highly valuable fungus is widely recommended by the traditional Chinese medicine practitioners for food intoxication, vomiting, and poisoning [1]. In addition, it was shown effective to improve liver and stomach immunity [2]. Due to its medicinal value and scarcity in nature, excessive forestry cutting down of Cinnamomum kanehirai is prohibited by the Taiwanese government [3].

\footnotetext{
* Correspondence: s9362284@hkbu.edu.hk

* Correspondence: s9362284@hkbu.edu.hk Kong SAR, China
} Kong SAR, China

After the success in mass production of AC by artificial cultivation, a series of health supplements formulated from $\mathrm{AC}$ has been launched with high market value [3], and are increasingly popular in the Taiwan, Japan, and other Asian regions. Counterfeit over-thecounter AC products have been found and reported. However, there is no reliable quality assessment method to evaluate the AC-based health supplements.

Currently, information regarding the bioactivity, pharmacology and, in particular, the chemical composition of AC is scarce [3-5]. Most AC research has been focused on the crude isolated fractions, which are subjected to pharmacological screening or therapeutically evaluation [6-12]. Recent research into the bioactivity of $\mathrm{AC}$, in treating liver diseases [13] with its biochemical mechanisms derived.

Triterpenoids and polysaccharides have been the focus of numerous AC studies due to their well-known pharmacological activities $[7,12,14]$. In mycelial AC, these 
bioactive chemicals include amino acids [14,15]; lipopolysaccharides [16]; nucleosides and nucleobases such as adenosine, cordycepin, cytidine, and thymine $[10,11,17,18]$; maleic acid and succinic acid derivatives $[6,19,20]$; benzenoids [21]; phenol and tocopherols [8,22]; 5'-nucleotides [14]; and diterpenes [23].

No chemical standardization or quality evaluation methods have been established for AC. As widely used in the quality control practices for other herbs, chromatographic fingerprinting is simple and useful. Thus, this study aims to identify the full profile fingerprint of nucleosides and nucleobases in mycelial AC by using high-performance liquid chromatography coupled with diode array detector and mass spectrometry (HPLCDAD-ESI-MS) and to assess the quality of two commercial mycelial AC products.

\section{Methods \\ Plant}

Powdered mycelium and an intact fruiting body of AC were supplied by GeneFerm Biotechnology Co. Ltd of Taiwan. Samples of two over-the-counter mycelial products were purchased from a Taiwanese commercial vendor (Hung-An Pharmacy). The crude herb was morphologically and microscopically authenticated by pharmacognosist Zhongzhen Zhao at Hong Kong Baptist University. The fruiting body was cut into small pieces and ground to powder. The powder of the samples was used for analysis.

Instrumentation

A Waters 2695 series HPLC system (Waters, USA) coupled with a Waters 2996 PDA (Waters, USA) was used. The column configuration consisted of a reverse phase $\mathrm{C}_{18} \mathrm{AQ}$ column (Alltech, Alltima, $250 \times 4.6 \mathrm{~mm}$, $5 \mu \mathrm{m}$ ) and an Econosphere $\mathrm{C}_{18}$ guard column (Alltech, Alltima, $7.5 \times 4.6 \mathrm{~mm}$ ). The mobile phase consisted of deionized water $(\mathrm{A})$, and methanol $(\mathrm{B})$ using the gradient program as follows: 0-15 minutes, $0 \% \mathrm{~B} ; 15-20 \mathrm{~min}$ utes, $0-2 \% \mathrm{~B}$; $20-30$ minutes, $2-15 \% \mathrm{~B}$; 30-40 minutes, $15-35 \%$ B; $40-50$ minutes, $35-60 \%$ B; 50-65 minutes, 60 $70 \%$ B; 65-80 minutes, 70-85\% B; 80-95 minutes, 85 $100 \% \mathrm{~B}$; and $95-115$ minutes, $100 \% \mathrm{~B}$. The flow rate was $1.0 \mathrm{ml}$ per minute with an injection volume of $10 \mu \mathrm{l}$. The column was maintained at room temperature of $25^{\circ}$ $\mathrm{C}$, and the re-equilibration time of the column was maintained as five minutes before another injection. The PDA detector (Waters, USA) was set at the optimum wavelength of $260 \mathrm{~nm}$.

An Agilent 1100 series HPLC-DAD system (Agilent, USA) coupled with an ion trap mass spectrometry detector was used. The system was equipped with an electrospray ionization (ESI) source and an ion trap analyzer for UV and MS data acquisition. A reverse phase $\mathrm{C}_{18}$ AQ (Alltech, Alltima, $250 \times 4.6 \mathrm{~mm}, 5 \mu \mathrm{m}$ ) column with a $300 \mathrm{SB}-\mathrm{C}_{18}$ (Zorbax, $\left.12.5 \times 4.6 \mathrm{~mm}, 5 \mu \mathrm{m}\right)$ guard column was used. The signals from the mass detector were recorded and analyzed by Bruker Daltonics data analysis software (Bruker, USA). The mobile phase for the qualitative analysis of the samples consisted of 5 $\mathrm{mM}$ ammonium acetate in deionized water, $\mathrm{pH} 6.79$ (A), and methanol (B) by using the gradient program as follows: 0-5 minutes, $0 \% \mathrm{~B}$; $5-10$ minutes, $0-2 \% \mathrm{~B} ; 10-20$ minutes, $2 \%$ B; $20-25$ minutes, $2-4 \%$ B; $25-30$ minutes, 4-6\% B; 30-40 minutes, $6-15 \% \mathrm{~B}$; and $40-60$ minutes, $15-100 \% \mathrm{~B}$. The flow rate was $1.0 \mathrm{ml}$ per minute with an injection volume of $20 \mu \mathrm{l}$. The column was maintained at room temperature $\left(25^{\circ} \mathrm{C}\right)$. The ESI-MS spectra were acquired in both positive and negative ion modes and compared on their relative sensitivities on the target compounds of interest. The capillary voltage was set at $-4 \mathrm{kV}$. The full scan mass spectra were obtained from a range of $\mathrm{m} / \mathrm{z}$ from 50 to 400 . The nebulizer pressure was at $30 \mathrm{psi}$. The flow rate of dry gas was maintained at 6 litres per minute. Dry gas temperature was maintained at $350^{\circ} \mathrm{C}$, and the collision energy was set at $2 \mathrm{eV}$. Solvents and chemicals

HPLC-grade solvents including methanol, acetonitrile, analytical grade chemicals including phosphoric acid, acetic acid, sodium hydroxide, and ammonium acetate, and deionized water generated from an Milli- $Q$ water system were used for the preparation of mobile phases. Chemical standards of cytosine, cytidine, adenosine, adenine, inosine, guanine, cordycepin, uracil, and uridine (>99\%; Sigma) were available for the identification of compounds in the samples.

\section{Sample preparation and chromatography}

For the chromatographic profile of water extracts, $0.1 \mathrm{~g}$ of the sample was accurately weighed and extracted in 2 $\mathrm{ml}$ of Milli-Q water under ultrasonication for $45 \mathrm{~min}$ utes at room temperature. The supernatant was then filtered through a $0.45 \mu \mathrm{m}$ Millipore filter before injecting $10 \mu \mathrm{l}$ into the HPLC. For the chromatographic fingerprint, $0.1 \mathrm{~g}$ of the sample was accurately weighed and extracted in $10 \mathrm{ml}$ of methanol in a conical flask under ultrasonication for 45 minutes at room temperature. The supernatant was then filtered, dried, and reconstituted into $2 \mathrm{ml}$ of methanol and water (85:15). The reconstituted solution was then filtered before HPLC injection.

\section{Results and discussion}

Nucleosides and nucleobases as major components of water extract

The chemical components in the water-soluble fraction were characterized by comparison with authentic chemical markers and LC-ESI-MS for structural elucidation. Experimental parameters were systematically adjusted to obtain the maximum number of extractable chemical 
compounds for a comprehensive chemical profile. Two major chemical groups, namely polysaccharides $[2,7,12,24,25]$ and 5'-nucleotides [14], together with nucleosides and nucleobases such as adenosine, cordycepin, cytidine, and thymine, were identified in the water extract of AC. As our previous study on Ganoderma lucidum, which is closely related fungus in taxonomy [3-5] and therapeutic value [26-28], also identified nucleosides and nucleobases as the major components [26], the full profile of nucleosides and nucleobases in $\mathrm{AC}$ can be useful in developing a fingerprint.

An extensive determination of the nucleoside and nucleobase profiles in the water extract of $A C$ was therefore conducted. Ten nucleosides or nucleobases (namely, cytidine, cytosine, adenine, adenosine, uridine, uracil, guanine, inosine, guanosine, and 2'-deoxyadenosine) were identified in the mycelia AC (Figure 1). Based on the ESI-MS, the molecular and product ions were observed in the forms of $[\mathrm{M}+\mathrm{H}]^{+},[\mathrm{M}+\mathrm{K}]^{+}$, and $[\mathrm{M}+\mathrm{Na}]$ ${ }^{+}$. Positive scan mode was chosen because of nucleosides and nucleobases are basic compounds and are more likely to be ionized with cations such as $\mathrm{H}^{+}, \mathrm{K}^{+}$, and $\mathrm{Na}$ ${ }^{+}$, thus facilitating the ESI-MS detection. Figure 2 shows the chromatographic profile of the water extract of mycelial AC.

\section{Comprehensive chemical profile of $A C$}

The appropriate solvent should be used to extract as many groups of representative chemical classes and compounds as possible to depict the chemical profile of a medicinal material. Methanol and $n$-hexane were employed for extracting compounds from mycelial and fruiting body AC $[18,21]$. In the present study, five solvents of different polarities (water, methanol, ethanol, chloroform, and n-hexane) were evaluated with regard to their extraction efficiency. We found that methanol was able to extract most chemical compounds. This solvent was chosen to maximize the number of compounds extracted from our AC samples.

\section{HPLC-DAD chromatographic fingerprint}

To ensure proper elution and separation of all characteristic compounds, polarities and $\mathrm{pH}$ of mobile phases were tested. The organic component of the mobile phase was alternated between methanol and acetonitrile. As the present $5 \mathrm{mM}$ ammonium acetate and methanol offer a basic aqueous environment for the analytes, an acidic counterpart of aqueous mobile phase with $0.1 \%$

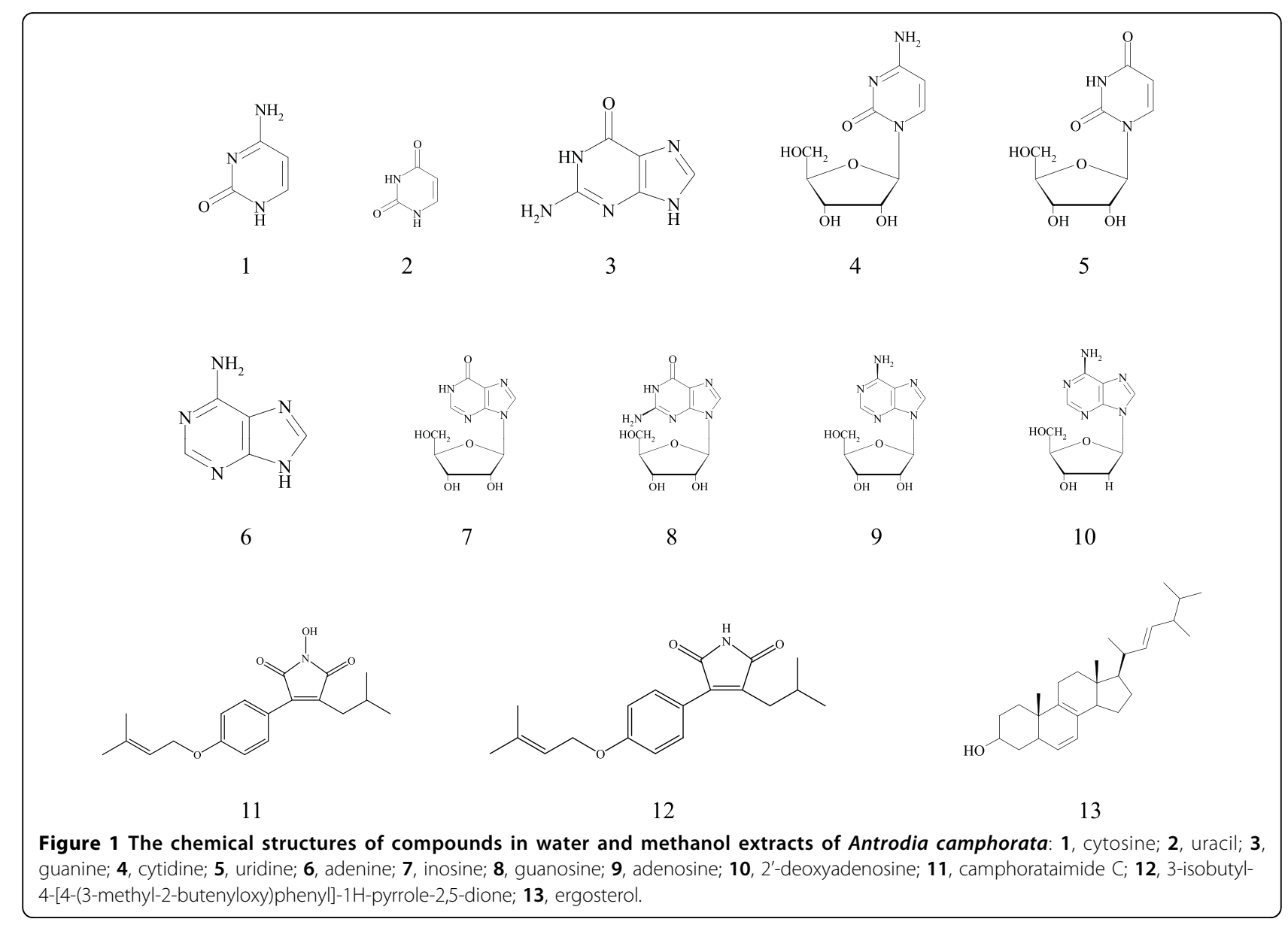




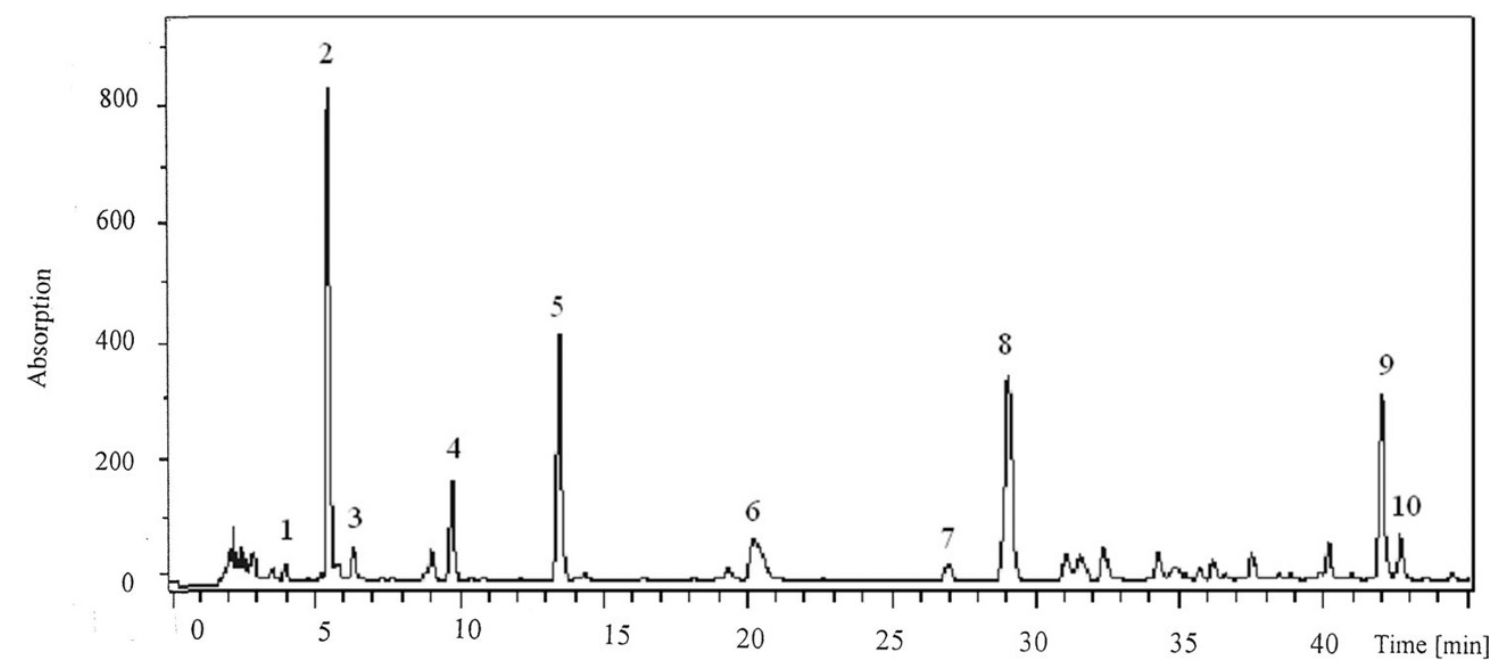

Figure 2 The HPLC-DAD chemical profile of the water extract of mycelial Antrodia camphorata: 1, cytosine; 2, uracil; 3, guanine; 4, cytidine; $\mathbf{5}$, uridine; $\mathbf{6}$, adenine; $\mathbf{7}$, inosine; $\mathbf{8}$, guanosine; $\mathbf{9}$, adenosine; $\mathbf{1 0}$, 2'-deoxyadenosine.

phosphoric acid in deionized water, $\mathrm{pH} 2.19$ and methanol was tested. In addition, a neutral aqueous mobile phase of deionized water and methanol was also tested. The use of neutral aqueous mobile phase showed more peaks but at the expense of peak shape and symmetry. Methanol is the best choice of organic components to facilitate elution of ergosterol, which is only compatible with solvents of lower polarity.

\section{Method validation}

To verify column performance and appropriateness of the chromatographic conditions, the number of theoretical plates, selectivity, resolution and peak symmetry values were determined as the indicators of separation efficiency. Resolution values were all higher than 1.5, which indicates good separation. Six replicate injections of a sample solution were performed to assess the precision of the methanol. The relative standard deviation (RSD) of relative retention time and relative peak area were less than $0.64 \%$ and $4.07 \%$, respectively. Another six independently prepared samples were assessed for the repeatability of the method. The RSD of relative retention time and relative peak area were $0.77 \%$ and $6.89 \%$, respectively. The sample stability was determined by three repetitive injections of a sample solution after three days of storage at room temperature. The RSD of relative retention time and relative peak area were $0.67 \%$ and $7.45 \%$, respectively.

\section{Qualitative chromatographic fingerprint}

The full profile of nucleosides and nucleobases was initially identified by matching the retention times and UV absorption profiles with respect to standards and was confirmed using ESI-MS. In total, ten compounds were identified in the water extract of mycelia AC. However, adenine, cytosine, and cytidine were not found when assessed using this new chromatographic condition, likely because their solubilities in the aqueous component of the mobile phase render poor column retention. Due to the bulky structures of these compounds (Figure 1), a specific extraction solvent and mobile phase were required for their coextraction and elution along with other compounds in the fingerprint. Our repeated trials for an optimal extraction showed that $100 \%$ methanol is the only choice capable of coextraction and elution. A gradient with $100 \%$ methanol was therefore adopted. In this way, different chemical compounds of various polarities are presented within the same chromatographic window despite the total elution time of all compounds lasting 120 minutes. Figure 3 shows the chromatographic fingerprint of methanol extract of mycelial AC. Preliminary application of mycelial AC chromatographic fingerprint

Two over-the-counter products that claimed consisted of mycelial AC were purchased from the Taiwanese market for our preliminary quality assessment. Figure 4 shows the superimposed chromatograms of methanol extract of the two commercial products in comparison to our reference fingerprint. The two commercial mycelial products possess very similar fingerprints, but these fingerprints are distinctively different from our established reference fingerprint of mycelial AC. From our morphological observation and confirmed by microscopic authentication during the species authentication stage, the powder in capsules are likely dried extracts rather than crude herbal material. The presence of additional possible herbal components other than those declared in the product package may also explain the difference in their derived fingerprints.

Moreover, the chemical compositions of mycelia and fruiting bodies have never been compared. The use of 


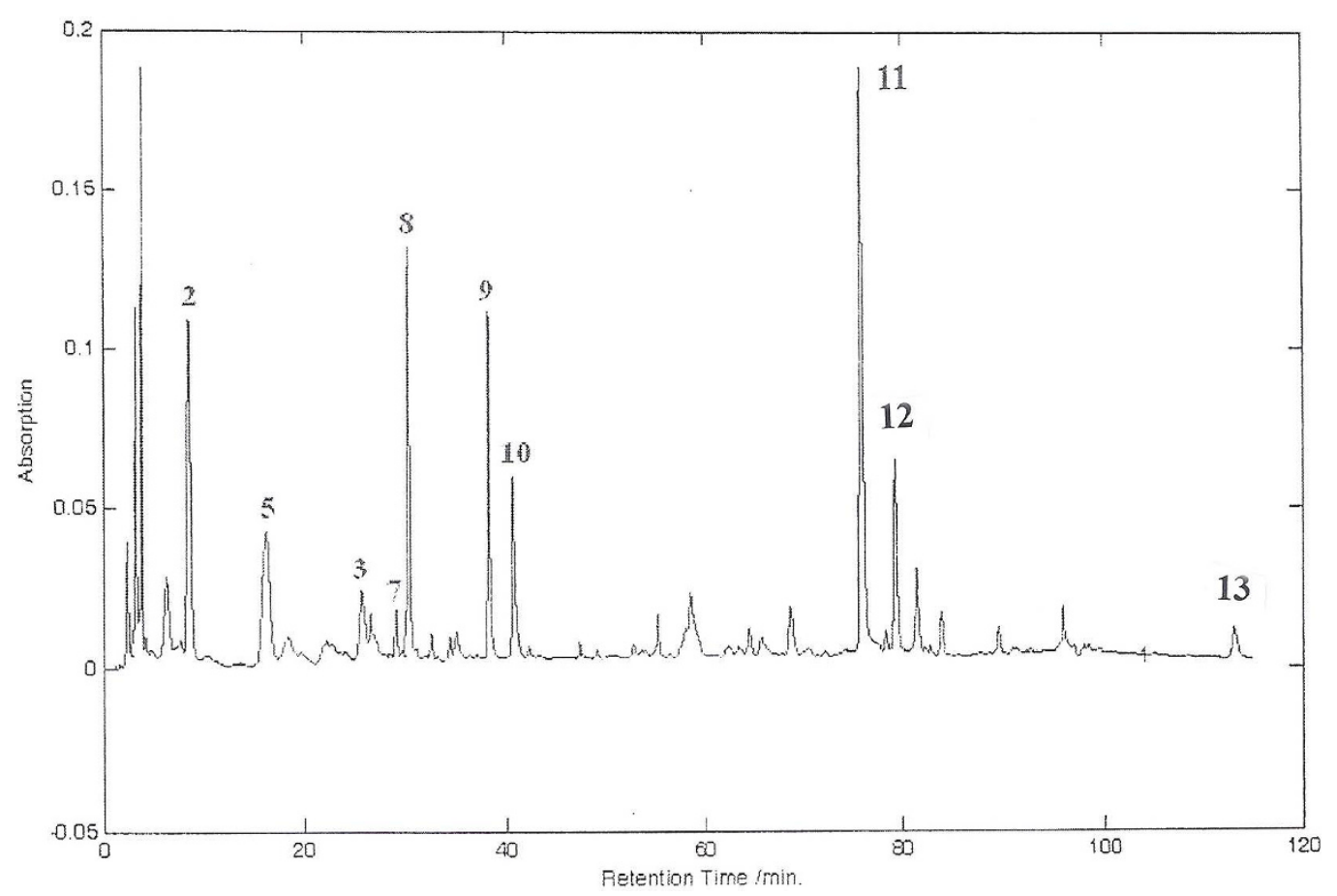

Figure 3 The established HPLC-DAD fingerprint of methanol extract of mycelial Antrodia camphorata: 2, uracil; 3, guanine; 5, uridine; 7, inosine; $\mathbf{8}$, guanosine; $\mathbf{9}$, adenosine; 10, 2'-deoxyadenosine; $\mathbf{1 1}$, camphorataimide C; $\mathbf{1 2}$, 3-isobutyl-4-[4-(3-methyl-2-butenyloxy)phenyl]-1H-pyrrole2,5-dione; 13, ergosterol.

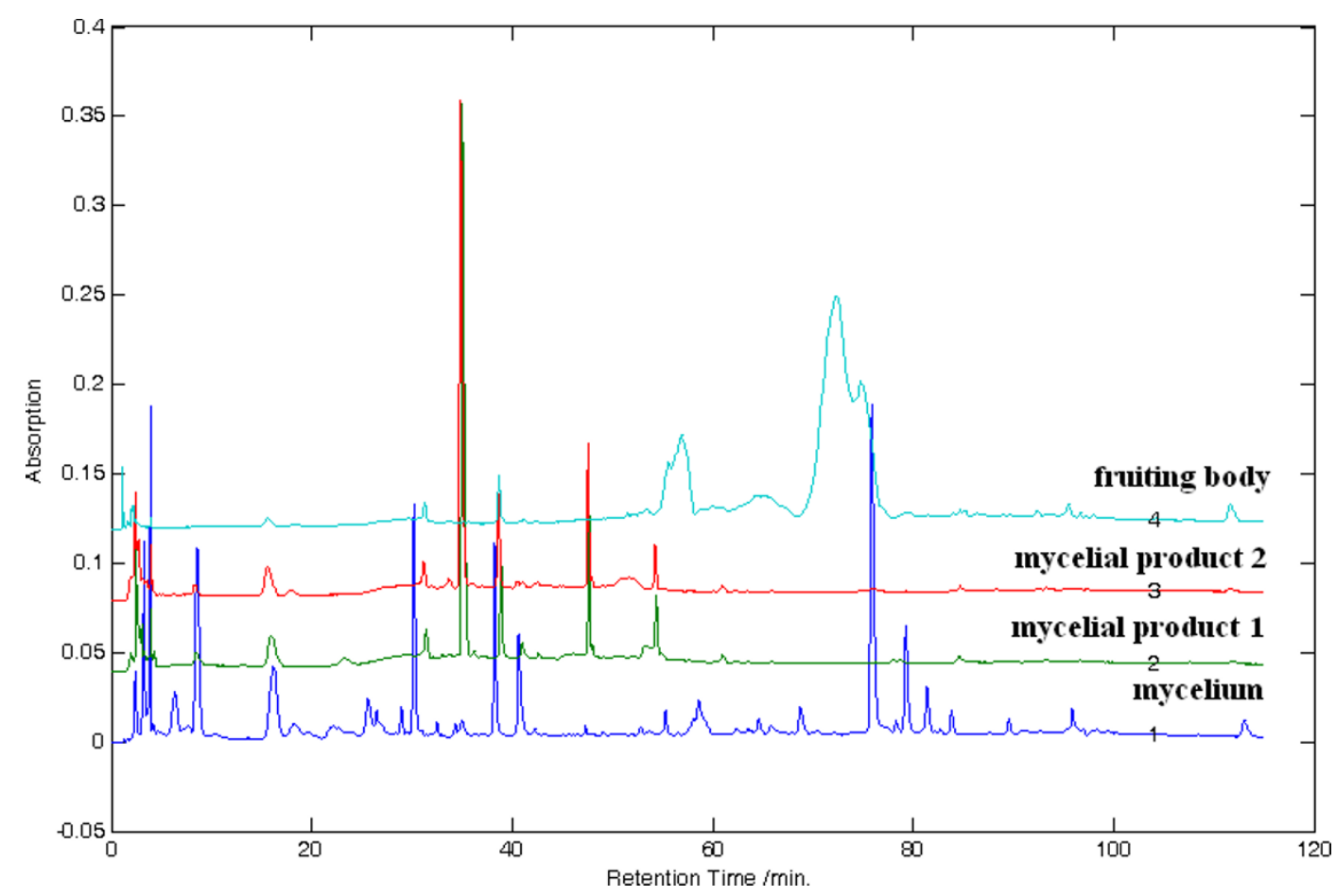

Figure 4 The superimposed HPLC-DAD chromatograms of methanol extracts of two commercial mycelial products: crude mycelium and crude fruiting body of Antrodia camphorata. For the sake of clarity, numbering of compounds is not shown. 
our chromatographic fingerprinting technique allowed a comparison of their chemical constituents. The fingerprint of the fruiting body part is distinctively different from that of the mycelium (Figure 4), suggesting there are different characteristic chemicals. In literatures, it suggested that the fruiting body is mainly composed of triterpenoids [29]. Therefore, specific reference chromatographic fingerprints should be used for independent quality control of the fruiting part of $\mathrm{AC}$.

\section{Conclusions}

This study provides the first chromatographic fingerprint to assess the quality of mycelial AC.

\section{Acknowledgements}

The authors would like to thank for the financial support of Faculty Research Grant [FRG/08-09/II-46] of the Hong Kong Baptist University. The generous donation of crude mycelial and fruiting bodies of Antrodia camphorata for the present study from GeneFerm Biotechnology Co. Ltd. of Taiwan is gratefully acknowledged.

\section{Authors' contributions}

Both authors took part in writing this manuscript. SSZ did the literatures review and all the experimental works. KSYL supervised on the project, advised and revised the manuscript. All authors read and approved the final version of the manuscript.

\section{Competing interests}

The authors declare that they have no competing interests.

Received: 30 October 2009

Accepted: 29 January 2010 Published: 29 January 2010

\section{References}

1. Hu O, Lian ZF, Zhang JY, Lu X: A review of the medicinal and health-care value: development and utilization of Antrodia camphorata. Subtrop Plant Sci 2006, 4:77-80.

2. Lee $\mathrm{HH}$, Huang RL, Chen CT, Chen HC, Hsu WC, Lu MK: Antrodia camphorata polysaccharides exhibit anti-hepatitis $B$ virus effects. FEMS Microbiol Lett 2002, 209:63-67.

3. Wu SH, Ryvarden L, Chang TT: Antrodia camphorata ("niu-chang-chih"), new combination of a medicinal fungus in Taiwan. Bot Bull Acad Sin 1997, 38:273-275.

4. Zang $\mathrm{M}, \mathrm{Su} \mathrm{CH}$ : Ganoderma comphoratum, a new taxon in genus ganoderma from Taiwan, PR China. Acta Bot Yunnanica 1990, 12(4):395-396.

5. Chang TT, Chou WN: Antrodia cinnamomea sp. nov. on Cinnamomum kanehirai in Taiwan. Mycol Res 1995, 99(6):756-758.

6. Nakamura N, Hirakawa A, Gao JJ, Kakuda H, Shiro M, Komatsu Y, Sheu CC, Hattori M: Five new maleic and succinic acid derivatives from the mycelium of Antrodia camphorata and their cytotoxic effects on LLC tumor cell line. J Nat Prod 2004, 67(1):46-48.

7. Chen CC, Liu YW, Ker YB, Wu YY, Lai EY, Chyau CC, Hseu TH, Peng RY: Chemical characterization and anti-inflammatory effect of polysaccharides fractionated from submerge-cultured Antrodia camphorata mycelia. J Agric Food Chem 2007, 55(13):5007-5012.

8. Mau JL, Huang PN, Huang SJ, Chen CC: Antioxidant properties of methanolic extracts from two kinds of Antrodia camphorata mycelia. Food Chem 2004, 86(1):25-31.

9. Huang NK, Cheng JJ, Lai WL, Lu MK: Antrodia camphorata prevents rat pheochromocytoma cells from serum deprivation-induced apoptosis. FEMS Microbiol Lett 2005, 244(1):213-219.

10. Lu MK, Cheng JJ, Lai WL, Lin YR, Huang NK: Adenosine as an active component of Antrodia cinnamomea that prevents rat PC12 cells from serum deprivation-induced apoptosis through the activation of adenosine $A_{2 A}$ receptors. Life Sci 2006, 79(3):252-258.
11. Lu MK, Cheng JJ, Lai WL, Lin YJ, Huang NK: Fermented Antrodia cinnamomea extract protects rat PC12 cells from serum deprivationinduced apoptosis: the role of the MAPK family. J Agric Food Chem 2008, 56(3):865-874.

12. Cheng JJ, Huang NK, Chang TT, Wang DL, Lu MK: Study for antiangiogenic activities of polysaccharides isolated from Antrodia cinnamomea in endothelial cells. Life Sci 2005, 76(26):3029-3042.

13. Ao ZH, Xu ZH, Lu ZM, Xu HY, Zhang XM, Dou WF: Niuchangchih (Antrodia camphorata) and its potential in treating liver diseases. J Ethnopharmacol 2008, 121(2):194-212.

14. Chang HL, Chao GR, Chen CC, Mau JL: Non-volatile taste components of Agaricus blazei, Antrodia camphorata and Cordyceps militaris mycelia. Food Chem 2001, 74(2):203-207.

15. Yue $Y Y$, Song AR, Tian XM, Wang F, Xu K: Composition analysis of amino acids in mycelia of Taiwanofungus formosanus. J Fungal Res 2006, 4(2):45-48.

16. Cheng JJ, Yang CJ, Cheng CH, Wang YT, Huang NK, Lu MK: Characterization and functional study of Antrodia camphorata lipopolysaccharide. J Agric Food Chem 2005, 53(2):469-474.

17. Wang GJ, Tseng HW, Chou CJ, Tsai TH, Chen CT, Lu MK: The vasorelaxation of Antrodia camphorata mycelia: involvement of endothelial $\mathrm{Ca}^{2+}-\mathrm{NO}-$ cGMP pathway. Life Sci 2003, 73(21):2769-2783.

18. Chang CY, Lue MY, Pan TM: Determination of adenosine, cordycepin and ergosterol contents in cultivated Antrodia camphorata by HPLC method. J Food Drug Anal 2005, 13(4):338-342.

19. Han HF, Hirakawa A, Zuo F, Nakamura N, Hattori M: Quantitative determination of maleic and succinic acid derivatives in the mycelium of Antrodia cinnamomea. J Trad Med 2006, 23(1):19-23.

20. Wu MD, Cheng MJ, Wang BC, Yech YJ, Lai JT, Kuo YH, Yuan GF, Chen IS: Maleimide and maleic anhydride derivatives from the mycelia of Antrodia cinnamomea and their nitric oxide inhibitory activities in macrophages. J Nat Prod 2008, 71(7):1258-1261.

21. Chen JJ, Lin WJ, Liao CH, Shieh PC: Anti-inflammatory benzenoids from Antrodia camphorata. J Nat Prod 2007, 70(6):989-992.

22. Huang SJ, Mau JL: Antioxidant properties of methanolic extracts from Antrodia camphorata with various doses of $\gamma$-irradiation. Food Chem 2007, 105(4):1702-1710

23. Chen CC, Shiao YJ, Lin RD, Shao YY, Lai MN, Lin CC, Ng LT, Kuo YH: Neuroprotective diterpenes from the fruiting body of Antrodia camphorata. J Nat Prod 2006, 69(4):689-691.

24. Hsu FL, Chou CJ, Chang YC, Chang TT, Lu MK: Promotion of hyphal growth and underlying chemical changes in Antrodia camphorata by host factors from Cinnamomum camphora. Int J Food Microbiol 2006, 106(1):32-38.

25. Chen SC, Lu MK, Cheng JJ, Wang DL: Antiangiogenic activities of polysaccharides isolated from medicinal fungi. FEMS Microbiol Lett 2005, 249(2):247-254.

26. Gao JL, Leung KSY, Wang YT, Lai CM, Li SP, Hu LF, Lu GH, Jiang ZH, Yu ZL: Qualitative and quantitative analyses of nucleosides and nucleobases in Ganoderma spp. by HPLC-DAD-MS. J Pharm Biomed Anal 2007, 44(3):807-811.

27. Fan H, Li SP, Xiang JJ, Lai CM, Yang FQ, Gao JL, Wang YT: Qualitative and quantitative determination of nucleosides, bases and their analogues in natural and cultured Cordyceps by pressurized liquid extraction and high performance liquid chromatography-electrospray ionization tandem mass spectrometry (HPLC-ESI-MS/MS). Anal Chim Acta 2006, 567(2):218-228.

28. Xie PS, Leung AY: Understanding the traditional aspect of Chinese medicine in order to achieve meaningful quality control of Chinese materia medica. J Chromatogr A 2009, 1216(11):1933-1940.

29. Shen CC, Kuo YC, Huang RL, Lin LC, Don MJ, Chang TT, Chou CJ: New ergostane and lanostane from Antrodia camphorata. J Chin Med 2003, 14(4):247-258.

doi:10.1186/1749-8546-5-4

Cite this article as: Zhao and Leung: Quality evaluation of mycelial Antrodia camphorata using high-performance liquid chromatography (HPLC) coupled with diode array detector and mass spectrometry (DAD-MS). Chinese Medicine 2010 5:4. 